\author{
MITSUBISHI ELECTRIC RESEARCH LABORATORIES \\ http://www.merl.com
}

\title{
Load Positioning in the Presence of Base Vibrations
}

\author{
Shilpiekandula, V.; Bortoff, S.A.; Barnwell, J.C.; El Rifai, K.
}

TR2012-049 June 2012

\begin{abstract}
A common problem in factory automation applications is one of lightly damped vibrations of base platforms used for supporting machine tools. Such vibrations arise from the inherent flexibility and low physical damping of the base platforms. Lightly damped base vibrations pose critical challenges in achieving performance specifications such as high tracking bandwidth or low settling times for the machine tools. Any external disturbances from the environment, or the servo torques applied to position the load, may excite the base. In this paper, we propose a switching control design for achieving the dual objectives of base vibration attenuation and fast load positioning. The proposed controller automatically switches between a vibration attenuation controller and a position tracking controller, with the switching implemented based on a state-dependent logic derived from a Lyapunov-based synthesis. The proposed controller is simple in its formulation, tuning, and implementation, and does not require a feedback sensor for measuring the base vibrations. To illustrate its effectiveness, experimental evaluations of the proposed controller and comparisons against a baseline linear controller are presented for a prototype hardware setup consisting of a servomotor-driven positioning stage mounted on a flexible base platform.
\end{abstract}

American Control Conference (ACC)

\footnotetext{
This work may not be copied or reproduced in whole or in part for any commercial purpose. Permission to copy in whole or in part without payment of fee is granted for nonprofit educational and research purposes provided that all such whole or partial copies include the following: a notice that such copying is by permission of Mitsubishi Electric Research Laboratories, Inc.; an acknowledgment of the authors and individual contributions to the work; and all applicable portions of the copyright notice. Copying, reproduction, or republishing for any other purpose shall require a license with payment of fee to Mitsubishi Electric Research Laboratories, Inc. All rights reserved.
}

Copyright (C) Mitsubishi Electric Research Laboratories, Inc., 2012

201 Broadway, Cambridge, Massachusetts 02139 



\title{
Load Positioning in the Presence of Base Vibrations
}

\author{
Vijay Shilpiekandula ${ }^{1}$, Scott A. Bortoff, John C. Barnwell III, Khalid El Rifai \\ Mitsubishi Electrical Research Laboratories, 201 Broadway Avenue, Cambridge MA 02139
}

\begin{abstract}
A common problem in factory automation applications is one of lightly damped vibrations of base platforms used for supporting machine tools. Such vibrations arise from the inherent flexibility and low physical damping of the base platforms. Lightly damped base vibrations pose critical challenges in achieving performance specifications such as high tracking bandwidth or low settling times for the machine tools. Any external disturbances from the environment, or the servo torques applied to position the load, may excite the base. In this paper, we propose a switching control design for achieving the dual objectives of base vibration attenuation and fast load positioning. The proposed controller automatically switches between a vibration attenuation controller and a position tracking controller, with the switching implemented based on a state-dependent logic derived from a Lyapunov-based synthesis. The proposed controller is simple in its formulation, tuning, and implementation, and does not require a feedback sensor for measuring the base vibrations. To illustrate its effectiveness, experimental evaluations of the proposed controller and comparisons against a baseline linear controller are presented for a prototype hardware setup consisting of a servomotor-driven positioning stage mounted on a flexible base platform.
\end{abstract}

\section{INTRODUCTION}

Increasing demands for high throughput and precision performance of machine tools dictate increasingly stringent requirements on machining tolerances and part-processing times [1]. Such requirements in turn necessitate high machine-tool tracking bandwidth and command-following accuracy [2]. In meeting such stringent performance requirements, and given the broad spectrum of disturbances on the factory floor, it becomes important to identify the source of the disturbances and effectively handle propagation of such disturbances from the source to the tool output.

A major source of disturbances in machine tools are lowfrequency vibrations of base platforms on which the machine tools are mounted. Such vibrations occur typically in the $1-10 \mathrm{~Hz}$ range and result from inherent flexibility of the base. A challenging aspect of such vibrations is that they are lightly damped, resulting in long-tailed transients and large settling times, which are detrimental to precision-positioning performance in the machine tools. We are interested in the attenuation of vibrations not only in the tool or load position output but also in the source, i.e. the base platform itself. Attenuating vibrations of the base platform is critical to minimizing cross-talk and propagation of disturbance between machine tool axes supported on the same base platform.

Many advanced command generation techniques, such as input shaping [3], and control techniques involving feed-

[1] Corresponding author: shilpiek@merl.com. forward and feedback ([9], [10], [13]) have been applied in the literature for minimizing residual vibrations. For the dual requirements of achieving acceptable positioning performance (high bandwidths or low settling times) as well as attenuation of base vibrations, we show here that a single linear controller is limited by inherent performance tradeoffs. In achieving multiple objectives, using hybrid controllers has been demonstrated to be a promising method to overcome inherent tradeoffs that cannot be overcome by linear control [11]. With this motivation, we propose a hybrid controller obtained by switching between two linear controllers: one dedicated to vibration attenuation and the other to load positioning for machine tools supported on flexible base platforms.

The rest of this report is organized as follows. Section II presents an overview of the problem of interest and discusses the target performance requirements to be met. A simple lumped-parameter model is presented in Section III. This model is used in the remainder of the report for control design. In Section IV, we discuss results from a robust analysis showing the fundamental tradeoffs in simultaneously achieving the dual objectives of vibration attenuation and load positioning with a single linear controller. The proposed solution for this problem, a novel control architecture using a control law that automates switching between separate controllers designed for each objective is discussed in Section V. A hardware prototype setup constructed for our experiments is described in Section VI. Herein, results from extensive hardware tests demonstrating effectiveness of the proposed controller are presented. A summary and directions for future research are detailed in Section VII.

\section{Problem Overview}

Fig. 1 shows a schematic diagram of the problem studied in this paper. An example set up of a machine tool axis: a motor-driven linear ballscrew stage, such as those described in [5] is shown as supported on a flexible base platform bolted to the ground. Such linear motion stages are components of most machine tool axes, and are hence the focus of our study.

The details of the selected machine tool axis are as follows. The mechanical components of the axis shown in Fig. 1 include (i) a rotary servomotor (ii) a rotary (relative) encoder integral to the motor, (iii) a linear ballscrew transmission connected to the motor shaft through a torque coupling, (iv) a load stage, and (v) a linear encoder mounted on the base platform for measuring the displacement of the load relative to the base platform. 
The target performance requirements are to (i) achieve fast settling times for smoothed step command inputs to the motor, and (ii) minimize vibrations (excited by command inputs and disturbances) in the load position, as well as the base position. The only actuator in the system is the rotary servomotor driving the machine tool axis. The only feedback sensor to be used in real-time for control design is the encoder on the motor or the load. No direct measurement (of position, velocity, or acceleration) of the base vibrations with another sensor should be used for control design. These constraints stem from practical considerations of the control design in a noisy factory floor environment for the case of a single base platform supporting many machine tool axes.

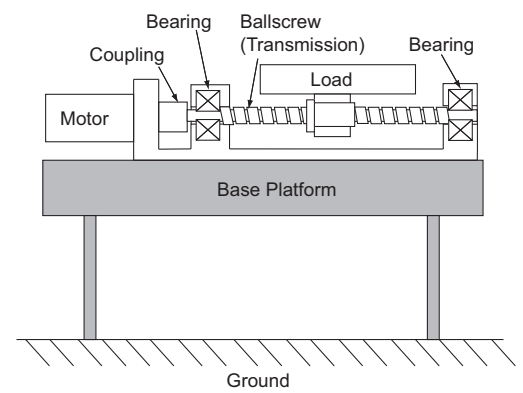

Fig. 1. Schematic diagram depicting a motor-driven linear ballscrew stage supported on a flexible base platform bolted to the ground.

\section{Modeling}

In this section, we present a simple lumped-parameter model for the system depicted in Fig. 1. Equations of motion and a state-space model are presented. The proposed lumped-parameter models adequately describe the dynamics of interest, and provide us with ample physical insights for observer and subsequent controller design in Section V. We refer the reader to detailed mechanical models of the ballscrew drive, such as those presented in [12], and models of flexible base platforms, such as those presented in [9], to build on the modeling presented in this section.

For the system of Fig. 1, consider the lumped-parameter model shown in Fig. 2. The fundamental vibration mode of

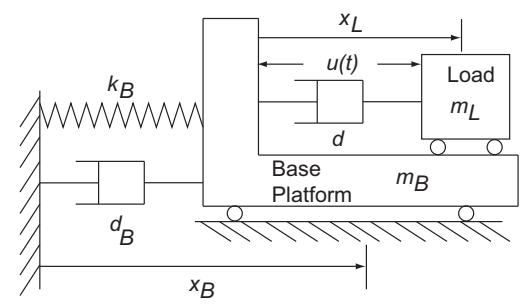

Fig. 2. Lumped parameter model of the mechanical system.

the base platform is depicted with a combination of a lumped mass in the rigid base platform mass $m_{B}$, a lumped stiffness in a linear spring $k_{B}$, and lumped physical damping in a linear viscous dashpot $d_{B}$.
The stator of the motor is attached rigidly to the base platform. The motor with the ballscrew transmission is modeled as a pure force source $u(t)$ that (i) drives the load (overcoming the physical damping in the ballscrew), and (ii) exerts an equal and opposite force on the base platform. For simplicity, a viscous linear dashpot $d_{L}$ is used to model the damping in the ballscrew. The stiffness of the ballscrew and associated elastic potential energy stored in it is neglected in this model. This is a reasonable simplification over low frequencies $(0-100 \mathrm{~Hz})$ for our choice of a compact ballscrew slide used in our experiments (see Section VI).

As denoted in Fig. 2, at any time $t$, for an absolute displacement $x_{B}(t)(m)$ of the base platform, a displacement $x_{L}(t)(m)$ of the load relative to the base platform, angular displacement $\theta(t)$ ( $r a d)$ of the motor, a pitch $p$ $(\mathrm{m} / \mathrm{rad})$ of the ballscrew, we define the state vector as

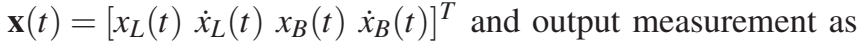
$y(t)=x_{L}(t)$, the displacement of the load as measured with a linear encoder, and obtain the corresponding state and output equations as follows:

$$
\begin{aligned}
\dot{\mathbf{x}}(t) & =A \mathbf{x}(t)+B u(t) \\
y(t) & =C \mathbf{x}(t)
\end{aligned}
$$

where

$$
\begin{gathered}
A=\left[\begin{array}{cccc}
0 & 1 & 0 & 0 \\
0 & -\frac{d_{L}}{m_{L}}-\frac{d_{L}}{m_{B}} & \frac{k_{B}}{m_{B}} & \frac{d_{B}}{m_{B}} \\
0 & 0 & 0 & 1 \\
0 & \frac{d_{L}}{m_{B}} & -\frac{k_{B}}{m_{B}} & -\frac{d_{B}}{m_{B}}
\end{array}\right] ; B=\left[\begin{array}{c}
0 \\
\left(\frac{1}{m_{L}}+\frac{1}{m_{B}}\right) \\
0 \\
-\frac{1}{m_{B}}
\end{array}\right] \\
C=\left[\begin{array}{llll}
1 & 0 & 0
\end{array}\right]
\end{gathered}
$$

\section{Performance Tradeoffs With Single Linear CONTROLler}

To gain physical insight into the inherent performance tradeoffs, let us consider the dynamics of the system under linear controllers dedicated for each objective. First, we examine the case of an example ideal reference-tracking controller and then an example ideal base vibration attenuation controller.

For load tracking, consider the control $u(t)=u_{r}(t)+$ $m_{L} \ddot{x}_{B}(t)$, where $u_{r}(t)$ is a reference-tracking controller, such as a PID controller. The resultant load and base dynamic equations are derived as follows:

$$
\begin{aligned}
m_{L} \ddot{x}_{L}(t) & =u_{r}(t)-d_{L} \dot{x}_{L}(t) \\
\left(m_{B}+m_{L}\right) \ddot{x}_{B}(t) & =-u_{r}(t)+d_{L} \dot{x}_{L}(t)-d_{B} \dot{x}_{B}(t)-k_{B} x_{B}(t)
\end{aligned}
$$

With the load tracking control proposed above, the load is isolated virtually from the base forces and hence, the desired ideal load tracking behavior can be achieved. However, the term $m_{L} \ddot{x}_{B}(t)$ adds to the moving mass of the base, hence the damping and the natural frequency of the base vibration mode are reduced. This will result in large base vibrations when the load is excited. Thus, the base dynamics are adversely affected by the ideal load tracking controller. 
For base vibration attenuation, consider the control $u(t)=$ $k_{D} \dot{x}_{B}(t)+d_{L} \dot{x}_{L}(t)$ aimed at increasing damping in the base and isolating it virtually from the load. The resultant load and base dynamic equations are as follows:

$$
\begin{aligned}
& m_{L} \ddot{x}_{L}(t)=\left\{\frac{m_{L}}{m_{B}} d_{B}+k_{D}\left(1+\frac{m_{L}}{m_{B}}\right)\right\} \dot{x}_{B}(t)+\frac{m_{L}}{m_{B}} k_{B} x_{B}(t) \\
& m_{B} \ddot{x}_{B}(t)=-\left(k_{D}+d_{B}\right) \dot{x}_{B}(t)-k_{B} x_{B}(t)
\end{aligned}
$$

With the base vibration attenuation control proposed above, the base dynamics are improved due to the increased damping. However, the load damping term in the load dynamics is cancelled out, thereby resulting in long settling times for the load and, hence, poor positioning performance.

From the above two cases, it follows that an ideal load positioning control compromises on base vibration attenuation, and an ideal base vibration attenuaton controller compromises on load positioning performance.

The inherent performance tradeoffs illustrated above can be studied using a robust control framework as well. To this end, a $\mathscr{H}_{\infty}$-synthesis procedure was adopted to generate a single linear controller that potentially should meet the objectives of both vibration attenuation and load positioning at fast settling times.

Fig. 3 shows the magnitude plot of the gain transfer function from disturbance to base vibration amplitude for increasing weight on the sensitivity. As the weights increase there is a corresponding decrease in the peak amplitude in the sensitivity magnitude plot. The corresponding effect (of increasing base vibration sensitivity weights) on the positioning error sensitivity transfer function is shown in Fig. 4.

For a single linear controller designed using the $\mathscr{H}_{\infty^{-}}$ synthesis, it is determined from Figs. 3 and 4 that for a $16 d B$ attenuation in the base vibration amplitude, there is a decrease in positioning bandwidth (and a corresponding increase in the settling time) by about a factor of 9 . Hence, we have shown that under the $\mathscr{H}_{\infty}$ framework, using a single linear controller results in a performance tradeoff: the more the base vibration attenuation, the smaller the positioning bandwidth, slower the system time response and longer the settling times.

\section{Proposed Controller}

\section{A. Control Architecture}

Fig. 5 shows the proposed control architecture including two controllers, one dedicated for vibration attenuation and another for position tracking. The system is commanded with a reference signal which can be prefiltered or input-shaped in the reference filter block. A switching function, which we will derive shortly, is calculated based on the present control input and full state (measured or reconstructed with an observer) and used to select a controller that maximizes the rate of decay of a physical energy-based Lyapunov function of the system.

The state and output equations Eqs. (1)-(2) for our lumpedparameter model of Fig. 2 represent a controllable and

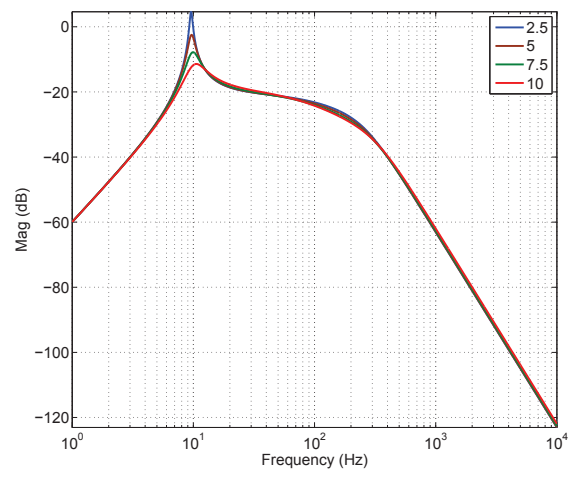

Fig. 3. Magnitude response, representing the gain from the disturbance to the base vibration amplitude, is shown for different weights chosen for the base vibration sensitivity transfer function.

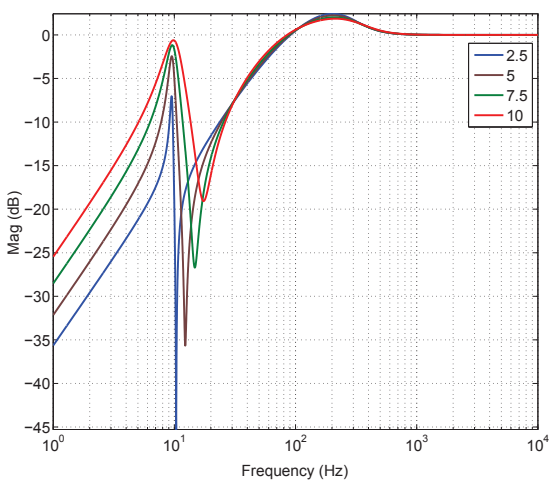

Fig. 4. Magnitude response of the error sensitivity transfer function, representing the gain from the disturbance to the positioning error, is shown for different weights chosen for the base vibration sensitivity transfer function.

observable system. In practice, there maybe higher order modes that we have neglected in our simple model. Here, we assume that such modes are stable, i.e. we assume an admissible and reachable system in our control design.

\section{B. Control Design}

For the system described in Section III, with state and output equations as given in Eq. (2), consider the control input $u(t)$ as defined below:

$$
u(t)=\alpha u_{b}(t)+(1-\alpha) u_{r}(t)
$$

where, $u_{b}(t)$ is a base vibration attenuation control input, and $u_{r}(t)$ is reference tracking control input, and $\alpha$ is a scalar that assumes a value of 0 or 1 at any time. The value of $\alpha$ is dictated by a switching function $f(t)$ as follows:

$$
\begin{array}{rlll}
\alpha & =0 & f(t) \geq 0 \\
& =1 & f(t)<0
\end{array}
$$

In other words, the reference tracking controller is selected at all times when the switching function $f(t) \leq 0$, and at other times, the base vibration attenuation controller 


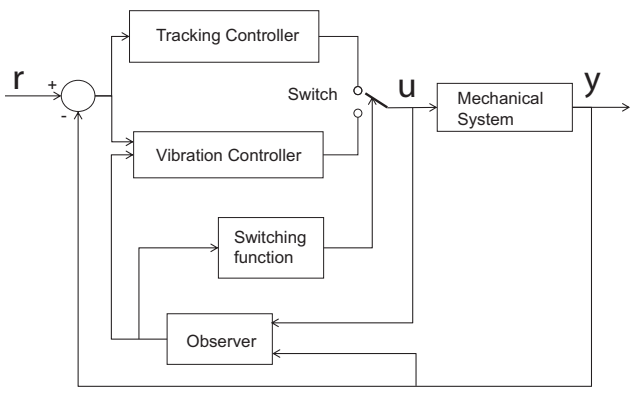

Fig. 5. Proposed control architecture proposed for simultaneous base vibration attenuation and precise position tracking.

is selected. Note that unlike approaches based on openloop time-switching, here the switching occurs in a statedependent manner, and closed-loop stability and performance requirements (settling time and vibration attenuation) dictate the switching times.

To address the given stability and performance requirements, it is essential to relate the function $f(t)$ to the full state (measured, or as in our case, reconstructed from an observer) of the system. In determining the switching function $f(t)$, while many choices exist for the controllers, we have adopted simple linear quadratic regulators [6] for this problem because of their ease of use and of tuning in real-time on a factory floor. With tunable weights on states and control inputs in user-defined horizon cost functions, linear quadratic regulators can be formulated to optimize the closed-loop system for the performance requirements of fast settling times and higher vibration attenuation. However, it should be noted that the proposed framework is amenable to inclusion of any type of control law for load positioning and base vibration attenuation. In what follows, we present a design for the switching function $f(t)$ and provide guarantees for stability of the closed-loop switched system.

1) Theorem: For the system given in Eqs. (1)-(2), and a control law as defined in Eqs. (7)-(9), the origin is globally exponentially stable [8] for the case of a switching function $f(t)$ defined as follows:

$$
\begin{aligned}
f(t)= & \mathbf{x}^{T}\left\{A^{T}\left(P_{r}-P_{b}\right)+\left(P_{r}-P_{b}\right) A\right. \\
& \left.-2\left(P_{r}-P_{b}\right) B R_{b}^{-1} B^{T} P_{b}\right\} \mathbf{x}
\end{aligned}
$$

where $A, B$ are the system matrices, and symmetric positive definite matrices $P_{r}$ and $P_{b}$ are solutions to the infinitehorizon Riccati equations given as follows:

$$
\begin{gathered}
A^{T} P_{r}+P_{r} A-P_{r} B R_{r}^{-1} B^{T} P_{r}+Q_{r}=0 \\
A^{T} P_{b}+P_{b} A-P_{b} B R_{b}^{-1} B^{T} P_{b}+Q_{b}=0
\end{gathered}
$$

where matrices $Q_{r} \geq 0, Q_{b} \geq 0$, are user-defined penalties on the state and $R_{r}>0, R_{b}>0$ are user-defined penalties on the control input.
In terms of the above-defined matrices, the , $u_{b}(\mathrm{t})$ is the vibration attenuation control signal, $u_{r}(\mathrm{t})$ is the positioning control signal, of Eq. (3) are given by:

$$
\begin{aligned}
& u_{b}(t)=-R_{b}^{-1} B^{T} P_{b} \mathbf{x}(t)=k_{b} \mathbf{x}(t) \\
& u_{r}(t)=-R_{r}^{-1} B^{T} P_{r} \mathbf{x}(t)=k_{r} \mathbf{x}(t)
\end{aligned}
$$

\section{Proof:}

We adopt a common Lyapunov function approach (see [4], [7], [14], and references therein) to construct our proof. Consider a common Lyapunov function defined as follows:

$$
V(t)=\mathbf{x}^{T}(t) P_{r} \mathbf{x}(t)>0
$$

Note that the choice of the common Lyapunov function is not unique. The reasoning behind our choice in Eq. (15) is that it represents the virtual energy of the closed-loop switched system, with $P_{r}$ as defined in Eq. (11). Using Eqs. (1), (2), and control law Eqs. (7)-(9), the corresponding rate of change of this function is given as follows:

$$
\begin{aligned}
\dot{V}(t)= & \alpha f(t)+\mathbf{x}^{T}\left[\alpha\left(-Q_{b}-P_{b} B R_{b}^{-1} B^{T} P_{b}\right)\right. \\
& \left.+(1-\alpha)\left(-Q_{r}-P_{r} B R_{r}^{-1} B^{T} P_{r}\right)\right] \mathbf{x}
\end{aligned}
$$

Given the switching law defined in Eqs. (8)-(9), it follows that in Eq. (16) the first term of $\alpha f(t) \leq 0$. Further, the second term in Eq. (16) is negative definite based on the definitions of the state and control penalties. Hence, it follows that $\dot{V}(t)<0$, and since $V(t)>0$, the origin is a globally asymptotically stable equilibrium point for the system [8].

To extend the stability analysis, it can be shown from Eq. (16) that $\dot{V}(t)$ is upper bounded as follows:

$$
\dot{V}(t) \leq-\mathbf{x}^{T}(t) Q_{u} \mathbf{x}(t)
$$

where

$$
\begin{aligned}
& Q_{u}= \\
& \operatorname{argmin}\left[\lambda_{\min }\left\{Q_{b}+P_{b} B R_{b}^{-1} B^{T} P_{b}, Q_{r}+P_{r} B R_{r}^{-1} B^{T} P_{r}\right\}\right]
\end{aligned}
$$

Using an eigen value analysis, the above inequalities further imply that:

$$
\dot{V}(t) \leq-\frac{\lambda_{\min }\left\{Q_{u}\right\}}{\lambda_{\max }\left\{P_{r}\right\}} V(t)
$$

and the corresponding inequality for the state reduces to:

$$
\|\mathbf{x}(t)\| \leq e^{\left[-\frac{\lambda_{\min }\left\{Q_{u}\right\}}{2 \lambda_{\max }\left\{P_{r}\right\}}\right]} \sqrt{\kappa\left\{P_{r}\right\}}\|\mathbf{x}(0)\|
$$

where and $\lambda_{\min }(\bullet)$ denotes the smallest eigen value, $\lambda_{\max }(\bullet)$ the largest eigen value and $\kappa(\bullet)$ denotes the condition number of the matrix $(\bullet)$. Since this inequality holds for all initial conditions $\mathbf{x}(0)$, the origin is an globally exponentially stable equilibrium point for the system, and the rate of convergence to the origin is $\frac{1}{2} \frac{\lambda_{\min }\left\{Q_{u}\right\}}{\lambda_{\max }\left\{P_{r}\right\}}$. This completes the proof of the theorem.

Note that the above theorem allows for stability analysis for perturbations in initial conditions on the state. For setpoint tracking problems, the above theorem can be extended to include an augmented state which includes tracking error 
as well. The physical interpretation of the presented theorem is as follows. The chosen common Lyapunov function represents a hyper-paraboloid centered around the origin in the state space. For any initial conditions of the system, the system trajectories converge to the origin at an exponential rate given by at least the rate of convergence derived above. The function $f(t)$ is switched between the two controllers to ensure that $\dot{V}(t)$ is negative and large in magnitude. In essence, we are increasing the decay rate of the virtual energy of the closed-loop switched system. For a vibration disturbance that dissipates stored potential energy of the flexible base, the switching function allows the appropriate motor torque to be applied to increase the rate of the dissipation. Similarly, for achieving load positioning, the switching function allows the control input to increase the rate of dissipation of the virtual potential energy associated with the tracking error.

\section{EXPERIMENTS AND RESULTS}

\section{A. Experimental Hardware Set up}

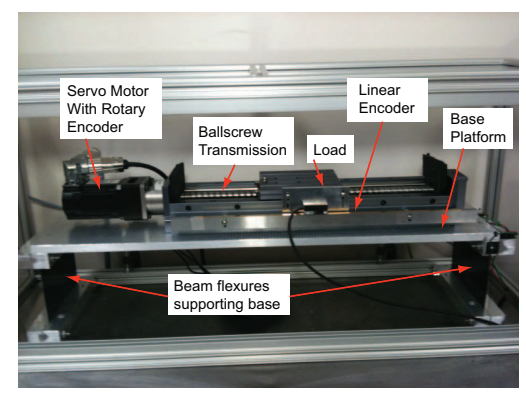

Fig. 6. Photograph of the base vibration hardware setup.

Fig. 6 shows the experimental test bed containing a base vibration platform controlled from a dSPACE DS1104 rapid prototyping control system. The base vibration system consists of a rotary servo motor driving a ball screw stage. The motor stator and the stage are fixed to a platform suspended on spring steel flexure beams designed for a fundamental vibration mode at about $10 \mathrm{~Hz}$. Two feedback sensors are provided in the system: one is the rotary encoder on the motor, and the other is a linear encoder mounted on the base platform and a readhead mounted on the moving stage. The linear encoder measures the displacement of the load with respect to the base. Assuming a stiff torque coupling and ballscrew transmission, we neglect the dynamics of the motion between the motor and the stage. The feedback control hence can use either the rotary motor encoder or the linear encoder mounted on the load.

Given the problem constraint that no feedback sensor is allowed for measuring the base vibrations for control design, a reduced order Luenberger observer was derived and realized in software to estimate the base position from measured signals. For monitoring base vibrations for testing purposes (and not for implementing feedback control) an accelerometer is mounted on the base to measure base accelerations.

\section{B. Results and Discussion}

The details of the control implementation and results from current experiments are as follows. The dSPACE DS1104 platform was used at execution rates of up to $40 \mathrm{kHz}$.

Fig. 7 shows the control signal for two separate experiments, one with a conventional PID controller and the other with the proposed hybrid control. Detailed time profiles of various control-related signals of interest are shown in Fig. 8. As shown in the figure, when the switching function $f(t)$ is positive or zero, the control switches to the load positioning controller; it switches to the base vibration controller otherwise. The switching is state-dependent and ensures closedloop stability.

As seen in the control signal in Fig 8, some chattering was observed at few periods of the traverse. This is undesirable since chattering may excite high-frequency dynamics (unmodeled structural modes). A low pass filter with a filter cut-off frequency at $200 \mathrm{~Hz}$ on the control input was found to be effective in minimizing chattering and significantly improving performance with respect to either control objectives.

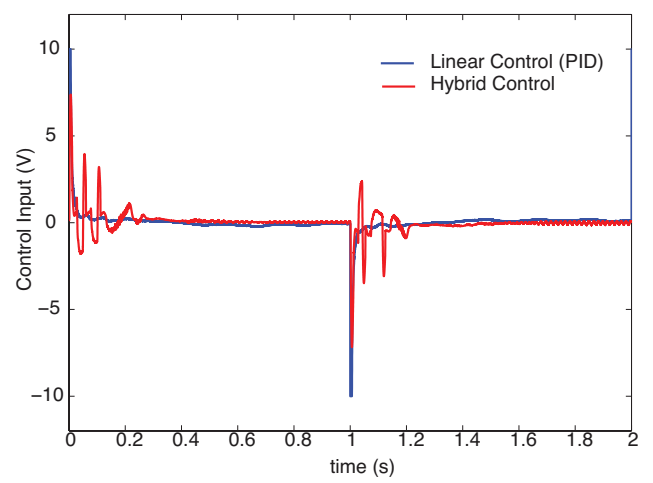

Fig. 7. Experimentally measured time profile of control input for (i) baseline PID controller and (ii) proposed hybrid controller.

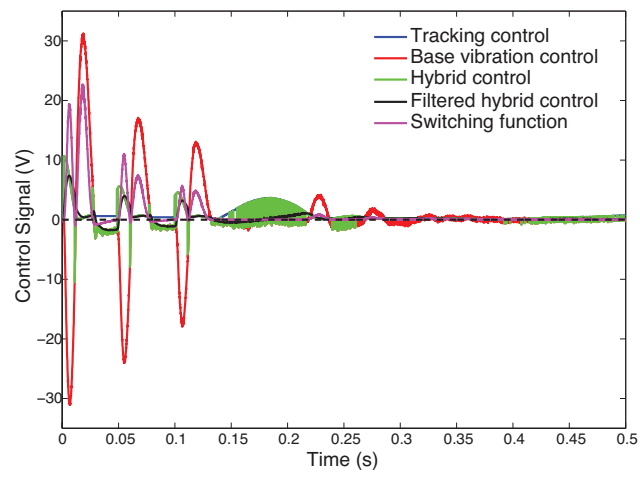

Fig. 8. Experimentally measured time profile of the control signal for the case of the proposed hybrid controller.

The performance of the system for base vibration attenuation is shown in Fig. 9. When compared to the conventional PID controller, the proposed controller improves base vibration attenuation by approximately $6 \mathrm{x}$ while reducing settling 
time by $2.5 x$, hence demonstrating the effectiveness of the proposed controller over conventional PID controllers. Testing for robustness to natural frequency of the fundamental natural frequency of the base is underway and will be the topic of a future work for our group.

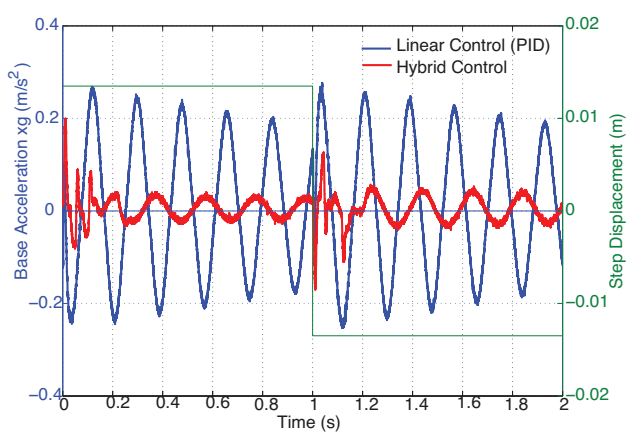

Fig. 9. Experimentally measured time profile of base vibration amplitude is shown for a square wave command input (shown in green).

In further extensions to the proposed controller, the switching control of Section V-B can be further generalized from the form in Eq. (15) to

$$
V(t)=\mathbf{x}^{T}(t) P \mathbf{x}(t)>0
$$

where $P$ is selected as a symmetric positive definite matrix that represents a virtual cost function for the system. For example, $P$ can be a diagonal matrix with elements selected to penalize base vibrations more than motor or load position. Furthermore, $P$ can also be selected to satisfy the following linear matrix inequality, which can be solved using efficient convex optimization algorithms:

$$
\left[\begin{array}{cc}
\left(A+B k_{r}\right)^{T} P+P\left(A+B k_{r}\right) & 0 \\
0 & -P
\end{array}\right]<0
$$

For the choice of $P$ in Eq. (27), one solution to the switching function $f(t)$ aimed at increasing the rate of decay of the Lyapunov function, is as follows

$$
\begin{aligned}
f(t)= & \mathbf{x}^{T}\left\{A^{T}\left(P-P_{b}\right)+\left(P-P_{b}\right) A\right. \\
& \left.-2\left(P-P_{b}\right) B R_{b}^{-1} B^{T} P_{b}\right\} \mathbf{x}
\end{aligned}
$$

Note that $P=P_{r}$ is one solution of Eq. (20) and Eq. (27), which when used in Eq. (22) reduces the switching function to the same as in Eq. (10).

Other extensions to the formulation can be in the form of constraints on control and state inputs. Recent advances in areas of constrained optimization and control, and especially, in model predictive control have shown that in fact even under constraints, the control inputs are indeed affine in the state. Further, the explicit linear quadratic regulator under input and state constraints have also been reported in the literature [16]. Using these advances in addressing constraint handling, the presented hybrid control framework can be extended to include state and control constraints as well.

\section{CONCLUSIONS}

In this paper, we have shown that using a single linear controller cannot facilitate achieving both vibration attenuation and load positioning performance requirements for mounting machine tools in a factory automation environment. The contributions of this paper are (i) a hybrid feedback controller developed using a common Lyapunov function approach, and the state-dependent switching control law (ii) a framework for addressing settling time and vibration attenuation requirements through easy-to-tune linear quadratic regulators. Further, a model-based dynamic reduced-order observer was constructed for base position, which is important when additional sensor(s) are not desired or cannot be implemented in the existing machine tools for real-time measurement of the base vibration. Implications for robustness and adaptation to uncertainties in base vibration resonances are subjects of future study.

\section{REFERENCES}

[1] Slocum AH, "Precision Machine Design," Englewood Cliffs, NJ, Prentice Hall, 1992.

[2] Srinivasan K and Tsao TC, "Machine Tool Feed Drives: A Survey of State of Art," Journal of Manufacturing Science and Engineering, vol. 119, pp. 743-748, 1997.

[3] W. Singhose, N. Singer, W. Seering, "Comparison of Command Shaping Methods for Reducing Residual Vibration," Proceedings of European Control, 1995.

[4] B. De Schutter, W.P.M.H. Heemels, A. Bemporad, "Modeling and Control of Hybrid Systems," Lecture Notes of the DISC Course, March 2003.

[5] Thomson Linear Motion Products, 203A West Rock Road Radford, VA 24141 USA. http://www.thomsonlinear.com/

[6] Anderson BDO, Moore JD, "Optimal Control: Linear Quadratic Methods," Mineola, NY, Dover Publications, 2007.

[7] E. Feron, "Quadratic stabilizability of switched systems via state and output feedback," Technical Report CICS-P-468, Center for intelligent control systems, Massachusetts Institute of Technology, Cambridge, 1996.

[8] Slotine JJ and Li W, “Applied Nonlinear Control," Prentice Hall, 1991.

[9] A. H. El-Sinawi, "Active Vibration Vsolation of a Vlexible Structure mounted on a Vibrating Elastic Base," Journal of Sound and Vibration, vol. 271 pp. 323-337, 2004.

[10] K. K. Miu, "A Low Cost, DC-Coupled Active Vibration Isolation System," M.S. Thesis, Department of Mechanical Engineering, Massachusetts Institute of Technology, Cambridge MA 02139.

[11] A. Feuer, G.C. Goodwin, M. Salgado, "Potential Benefits of Hybrid Control for Linear Time Invariant Plants," American Control Conference, Albuquerque NM, 1997.

[12] K.K. Varanasi and S.A. Nayfeh, "Modeling, Identification, and Control of Ballscrew Drives," American Society for Precision Engineering 16th Annual Meeting, v 25, p139-142, Crystal City, Virginia, 2001.

[13] Hara S, Kushida Y, Yamada Y, Fujimoto I, Morita Y, "Experimental Study on Vibration Control of Flexible Structures Taking Power Assisted Conveyance Into Account," In Proceedings of International Federation of Automatic Control Symposium on Mechatronics, Cambridge MA, USA, pp. 160-167, Sept 2010.

[14] Aravena JL, Devarakonda L, "Performance Driven Switching Control," in Proceedings of IEEE International Symposium on Industrial Electronics, Quebec, Canada, vol. 1, pp 31-36, 2006.

[15] dSPACE Inc., Alicia Alvin, 50131 Pontiac Trail, Wixom, MI, USA 48393-2020 USA. http://www.dspace.inc/

[16] Bemporad A, Morari M, Dua V, et al, "The explicit linear quadratic regulator for constrained systems," Automatica, vol. 38 (1), January 2000, pp. (3-20). 